\title{
ON THE MOBILITY AND EFFICIENCY OF MECHANICAL SYSTEMS*
}

\author{
Gershon WOLANSKY ${ }^{1}$
}

\begin{abstract}
It is shown that self-locomotion is possible for a body in Euclidian space, provided its dynamics corresponds to a non-quadratic Hamiltonian, and that the body contains at least 3 particles. The efficiency of the driver of such a system is defined. The existence of an optimal (most efficient) driver is proved.
\end{abstract}

Mathematics Subject Classification. 37J50, 37K25, 70H33.

Received February 8, 2006.

Published online July 20, 2007.

\section{INTRODUCTION}

This paper is motivated by a series of publications on the subject of self-locomotion, such as molecular motors and the flashing rachet. See $[2-4]$ and references therein.

An appealing geometric theory of self-locomotion was recently developed. Of particular interest is the "Baron Munchausen effect": In this classical book [8], the Baron lift himself (and his horse) out of the swamp by pulling his own hair. This, however, is possible only for the Baron. We all know that the center of mass of a system cannot be changed unless a force is applied from the outside. In [10], Wisdom argued that self locomotion is possible in a curved space. In [1] the authors expanded the geometrical theory behind self-locomotion (see also [6]). Their assumption is that a body (or system of mass points) is embedded in a homogeneous space equipped with a Riemannian metric. On such a space, self locomotion (or "swimming") is understood as a non-trivial isometry acting on the body as a result of a strain induced by a periodic deformation which the body apply upon itself. In other words, after the completion of a "swimming stroke" the body regains its original shape at a different location. A necessary condition for the self locomotion is the existence of nontrivial curvature associated with the underlying group of isometries. This implies that the Baron is lying, since he lives in Euclidian space where the group of translations is curvature free. On the other hand, a cat can turn itself upside down while falling in Euclidian space since the group of rotations is not curvature free. As argued by the authors of [1], this corresponds to the non-existence of a unique center of mass of the body in the underlying space.

The Riemannian structure is essential for the argument of [1]. The authors used it to separate the action of the isometry group from this of the deformations. It implies that the dynamics is determined by a Lagrangian

Keywords and phrases. Lagrangian mechanics, efficiency, self-locomotion.

* This research was supported by a grant from the Israel Science Foundation.

1 Department of mathematics, Haifa 32000, Israel; gershonw@math.technion.ac.il 
induced by the metric - in particular, a quadratic Lagrangian. See Section 4 below for further discussion of this point.

In this paper I consider self-locomotion form a somewhat different (perhaps more general) point of view and remove the limitation of quadratic Lagrangians. On the other hand, we stay in Euclidian space, where the group of translations acts transitively and the center of mass is defined in a unique way.

A mechanical system is represented by a shift-invariant Lagrangian which is symmetric under permutation of its coordinates. Some of the degrees of freedom are constrained to preserve their relative positions. The motion of the system is determined by its constant momentum vector. The rest state corresponds to a zero momentum vector.

By a deformation we mean a change in the relative positions which preserves their center of mass. A time periodic deformation is called a driver. The shift invariance of the system, along with the momentum vector, are still preserved, but the averaged velocity of the system may change. If such a change takes place then the driver is said to be a mobilizing driver for the prescribed momentum. Self-locomotion corresponds to a mobilizing driver which is initially at rest. The motion of such mobilizing driver corresponds to zero momentum.

There is a natural way to define the efficiency of a mobilizing driver in terms of the relation between the relative shift due the presence of the driver and the rate of energy invested in this driver. The question of existence of the best (most efficient) driver is a very natural one.

In Section 2 we pose the main assumptions and definitions - in particular, the definition of efficiency. The main theorem of this paper is presented and proved in Section 3. Here we specializes in homogeneous Lagrangian and prove the existence of a best (most efficient) driver. Finally, in Section 4 we discuss the relation between the assumptions of this paper and the geometrical theory of self-locomotion, as presented in [1].

\section{Description OF THE MODEL}

\subsection{Mechanical systems and drivers}

Consider the Lagrangian function $L(\dot{\boldsymbol{x}})$ on $\mathbb{R}^{n}$ where

$$
L: \mathbb{R}^{n} \rightarrow \mathbb{R}^{+} \cup\{0\} \text { is strictly convex and } L(\mathbf{0})=0,
$$

and, for any permutation $\pi:\{1, \ldots, n\} \rightarrow\{1, \ldots, n\}$

$$
L\left(v_{1}, v_{2}, \ldots, v_{n}\right)=L\left(v_{\pi(1)}, v_{\pi(2)}, \ldots, v_{\pi(n)}\right) .
$$

Let $p_{i}=\partial_{v_{i}} L$ the momentum associated with the $v_{i}$ and $\boldsymbol{p}:=\left(p_{1}, \ldots, p_{n}\right) \in \mathbb{R}^{n}$ any fixed momentum vector. The unconstrained orbit is just

$$
\boldsymbol{x}(t)=\dot{\boldsymbol{x}}\left(t-t_{0}\right)+\boldsymbol{x}_{0},
$$

where $\boldsymbol{x}_{0} \in \mathbb{R}^{n}$ and $\dot{\boldsymbol{x}}$ is uniquely determined by inverting

$$
\nabla_{\dot{\boldsymbol{x}}} L=\boldsymbol{p}
$$

The corresponding Hamiltonian function is defined, as usual, by the Legendre transform

$$
H(\boldsymbol{p}):=\sup _{\dot{\boldsymbol{x}} \in \mathbb{R}^{n}}[\dot{\boldsymbol{x}} \cdot \boldsymbol{p}-L(\dot{\boldsymbol{x}})] .
$$

Now, we introduce a constraint on this system as follows: The set $\{1, \ldots, n\}$ is decomposed into $N$ disjoint subsets (clusters) $I_{1} \ldots, I_{N}$ so $\cup_{1}^{N} I_{j}=\{1, \ldots, n\}$ and $I_{j} \cap I_{i}=\emptyset$ if $i \neq j$. We further assume that each cluster contains at least 2 elements. 
The position of the system at time $t$ can, thus, be represented by the $N$ vectors $\boldsymbol{x}_{I_{j}}=\left(x_{i} ; i \in I_{j}\right) \in \mathbb{R}^{I_{j}}$ for $j=1, \ldots, N$. The Lagrangian system can, in turn, be written as

$$
L=L\left(\dot{\boldsymbol{x}}_{I_{1}}, \ldots, \dot{\boldsymbol{x}}_{I_{N}}\right) .
$$

In each cluster $I_{j}$, the particles are constrained to move along a prescribed periodic orbit with respect to some point (say, the center of mass of the particles in this cluster). Let $\boldsymbol{y}_{I_{j}}(t)=\left(y_{i}(t), i \in I_{j}\right) \in \mathbb{R}^{I_{j}}$ be such a periodic orbit associated with the driver $j$, so

$$
\boldsymbol{y}_{I_{j}}(t)=\boldsymbol{y}_{I_{j}}(t+T) \quad \forall t \in \mathbb{R}, 1 \leq j \leq N .
$$

In addition we may assume that the "center of mass" of the each driver is at rest, that is

$$
\sum_{i \in I_{j}} y_{i}=0,1 \leq j \leq n
$$

We call such constraint $\boldsymbol{y}_{I_{j}}$ the $j$-driver. The position of $\boldsymbol{x}_{I_{j}}$ at time $t$ is, thus, determined by the single $\mathbb{R}$ valued function $X_{j}$ via

$$
\boldsymbol{x}_{I_{j}}(t)=\boldsymbol{y}_{I_{j}}(t)+X_{j}(t) \mathbf{1}_{j}
$$

where $\mathbf{1}_{j}:=(1, \ldots, 1) \in \mathbb{R}^{I_{j}}$. Let $\boldsymbol{X}:=\left(X_{1}, \ldots, X_{N}\right)$. Similarly, let $\boldsymbol{Y}:=\left(\boldsymbol{y}_{I_{1}}, \ldots, \boldsymbol{y}_{I_{N}}\right) \in \prod_{j=1}^{N} \mathbb{R}^{I_{j}}$. The constrained Lagrangian is now the function $\hat{L}: \mathbb{R}^{N} \times \prod_{j=1}^{N} \mathbb{R}^{I_{j}} \rightarrow \mathbb{R}$ given by

$$
\hat{L}(\dot{\boldsymbol{X}}, \dot{\boldsymbol{Y}}):=L\left(\dot{X}_{1} \mathbf{1}_{I_{1}}+\dot{\boldsymbol{y}}_{I_{1}} \ldots \dot{X}_{N} \mathbf{1}_{I_{N}}+\dot{\boldsymbol{y}}_{I_{N}}\right)
$$

and the associated momentum $\boldsymbol{P} \in \mathbb{R}^{N}$ defined by

$$
\boldsymbol{P}=\left(P_{1}, \ldots, P_{N}\right), P_{i}=\partial_{\dot{X}_{i}} \hat{L}(\dot{\boldsymbol{X}}, \dot{\boldsymbol{Y}}) .
$$

Using the Hamiltonian formulation we set the Hamiltonian $\hat{H}(\boldsymbol{P}, \dot{\boldsymbol{Y}})$ associated with the constrained system as

$$
\hat{H}: \mathbb{R}^{N} \times \prod_{j=1}^{N} \mathbb{R}^{I_{j}} \rightarrow \mathbb{R} ; \hat{H}(\boldsymbol{P} . \dot{\boldsymbol{Y}})=\sup _{\boldsymbol{W} \in \mathbb{R}^{N}}[\boldsymbol{W} \cdot \boldsymbol{P}-\hat{L}(\boldsymbol{W}, \dot{\boldsymbol{Y}})]
$$

The solution of the constrained system is now determined by any constant momentum vector $\boldsymbol{P} \in \mathbb{R}^{N}$ via

$$
\boldsymbol{X}(t)=\boldsymbol{X}(0)+\int_{0}^{t} \frac{\partial \hat{H}(\boldsymbol{P}, \dot{\boldsymbol{Y}})}{\partial \boldsymbol{P}} \mathrm{d} t
$$

and the displacement of the system after one period is given by

$$
\Delta_{\mathbf{Y}} \boldsymbol{X}:=\boldsymbol{X}(T)-\boldsymbol{X}(0)=\int_{0}^{T} \frac{\partial \hat{H}(\boldsymbol{P}, \dot{\boldsymbol{Y}})}{\partial \boldsymbol{P}} \mathrm{d} t .
$$

If all drivers $\dot{\boldsymbol{y}}_{I_{j}}$ are "turned off", so $\dot{\boldsymbol{Y}}=0$, then the respective displacement is given by

$$
\Delta_{\mathbf{0}} \boldsymbol{X}:=T \frac{\partial \hat{H}(\boldsymbol{P}, 0)}{\partial \boldsymbol{P}} .
$$


The difference

$$
\Delta_{Y} X-\Delta_{0} X
$$

is some measure of the mobility of the driver $\boldsymbol{Y}$.

Example. If $\boldsymbol{P}=0$ and the driver is turned off $(\dot{\boldsymbol{Y}}=0)$ then the system is at rest $(\dot{\boldsymbol{X}}=0)$. In particular, there is no displacement after a period $T\left(\Delta_{0} \boldsymbol{X}=0\right)$. If the driver is "turned on" at the same value $\boldsymbol{P}=0$, the displacement may be nonzero. In this case

$$
\left.\Delta_{\mathbf{Y}} \boldsymbol{X}\right|_{\boldsymbol{P}=\mathbf{0}}=\int_{0}^{T} \frac{\partial \hat{H}(\mathbf{0}, \dot{\boldsymbol{Y}})}{\partial \boldsymbol{P}} \mathrm{d} t \neq 0
$$

and the system is self-locomotive.

Definition 2.1. The rate of energy spent by the drivers $\boldsymbol{Y}$ for a prescribed $\boldsymbol{P} \in \mathbb{R}^{N}$ is

$$
S(\boldsymbol{P}, \boldsymbol{Y}):=-\frac{1}{T} \int_{0}^{T}[\hat{H}(\boldsymbol{P}, \dot{\boldsymbol{Y}})-\hat{H}(\boldsymbol{P}, \mathbf{0})] \mathrm{d} t .
$$

Remark 2.1. Definition 2.1 is rater natural since the Hamiltonian $\hat{H}(\boldsymbol{P}, \dot{\boldsymbol{Y}})$ is, physically, the energy state of the constrained system. If we reduce the energy state of the "free" system $\hat{H}(\boldsymbol{P}, \mathbf{0})$, then what is left is the energy executed by the constrains themselves. The minus sign applies for the energy spent on the constrains.

Lemma 2.1. The energy rate $S(\boldsymbol{P}, \boldsymbol{Y})$ is always non-negative.

Proof. By definition

$$
\hat{H}(\boldsymbol{P}, \dot{\boldsymbol{Y}}) \leq H\left(\mathbf{1}_{1} P_{1}, \ldots, \mathbf{1}_{N} P_{N}\right)
$$

where $\mathbf{1}_{j}$ is defined as in (2.5). Indeed,

$$
\begin{aligned}
& \hat{H}(\boldsymbol{P}, \dot{\boldsymbol{Y}})=\sup _{\dot{\boldsymbol{X}} \in \mathbb{R}^{N}}[\dot{\boldsymbol{X}}\left.\cdot \boldsymbol{P}-L\left(\mathbf{1}_{1} \dot{X}_{1}+\dot{\boldsymbol{y}}_{1}, \ldots, \mathbf{1}_{N} \dot{X}_{N}+\dot{\boldsymbol{y}}_{N}\right)\right] \\
&= \sup _{\dot{\boldsymbol{X}} \in \mathbb{R}^{N}}\left[\sum_{i=1}^{N}\left(\dot{X}_{i}+\sum_{j \in I_{i}} \dot{y}_{j}\right) P_{i}-L\left(\mathbf{1}_{1} \dot{X}_{1}+\dot{\boldsymbol{y}}_{1}, \ldots, \mathbf{1}_{N} \dot{X}_{N}+\dot{\boldsymbol{y}}_{N}\right)\right] \\
& \leq \sup _{\boldsymbol{W}:=\left(\boldsymbol{w}_{1}, \ldots, \boldsymbol{w}_{N}\right) ; \boldsymbol{w}_{j} \in \mathbb{R}^{I_{j}}}\left[\sum_{i=1}^{N}\left(\sum_{j \in I_{i}} w_{j}\right) P_{i}-L\left(\boldsymbol{w}_{1}, \ldots, \boldsymbol{w}_{N}\right)\right] \\
&=H\left(P_{1} \mathbf{1}_{1}, \ldots, P_{N} \mathbf{1}_{N}\right)
\end{aligned}
$$

where the second equality in (2.10) follows from (2.4). Finally, the definition of $\hat{H}(\boldsymbol{P}, \mathbf{0})$ implied by (2.7) and the symmetry of $L$ under permutations (2.2) yields

$$
H\left(P_{1} \mathbf{1}_{1}, \ldots, P_{N} \mathbf{1}_{N}\right)=\hat{H}(\boldsymbol{P}, \mathbf{0}) .
$$

In order to obtain a good notion of efficiency, the mobility vector (2.9) should be scaled against the energy rate $S(\boldsymbol{P}, \boldsymbol{Y})$. However, $(2.9)$ represents a vector while $S(\boldsymbol{P}, \boldsymbol{Y})$ is a scalar. In order to compare the two, we replace (2.9) by an equivalent, scalar measure which have the same scaling as the energy rate (see Sect. 3):

Definition 2.2. The driver $\boldsymbol{Y}$ is said to be $\boldsymbol{P}$-mobilized if

$$
\Delta(\boldsymbol{P}, \boldsymbol{Y}):=\hat{L}\left(\frac{\Delta_{\boldsymbol{Y}} \boldsymbol{X}}{T}, \mathbf{0}\right)-\hat{L}\left(\frac{\Delta_{\mathbf{0}} \boldsymbol{X}}{T}, \mathbf{0}\right)-T^{-1} \boldsymbol{P} \cdot\left[\Delta_{\boldsymbol{Y}} \boldsymbol{X}-\Delta_{\mathbf{0}} \boldsymbol{X}\right] .
$$


In particular, it is self locomotive if $\Delta(0, \boldsymbol{Y})>0$.

Lemma 2.2. For any $\boldsymbol{P} \in \mathbb{R}^{N}, \Delta(\boldsymbol{P}, \boldsymbol{Y}) \geq 0$. Moreover, $\Delta(\boldsymbol{P}, \boldsymbol{Y})>0$ if and only if $\Delta_{\boldsymbol{Y}} \boldsymbol{X}-\Delta_{0} \boldsymbol{X} \neq 0$.

Proof. By (2.8) it follows that

$$
T^{-1} \boldsymbol{P} \cdot \Delta_{\mathbf{0}} \boldsymbol{X}-\hat{L}\left(\frac{\Delta_{\mathbf{0}} \boldsymbol{X}}{T}, \mathbf{0}\right)=\boldsymbol{P} \cdot \frac{\partial \hat{H}(\boldsymbol{P}, \mathbf{0})}{\partial \boldsymbol{P}}-\hat{L}\left(\frac{\partial \hat{H}(\boldsymbol{P}, \mathbf{0})}{\partial \boldsymbol{P}}, \mathbf{0}\right)=\hat{H}(\boldsymbol{P}, \mathbf{0}) .
$$

By (2.7) it follows that

$$
\hat{H}(\boldsymbol{P}, \mathbf{0}) \geq \boldsymbol{P} \cdot \frac{\Delta_{\boldsymbol{Y}} \boldsymbol{X}}{T}-\hat{L}\left(\frac{\Delta_{\boldsymbol{Y}} \boldsymbol{X}}{T}, \mathbf{0}\right),
$$

and the inequality is strict unless $\Delta_{\boldsymbol{Y}} \boldsymbol{X}=\Delta_{0} \boldsymbol{X}$ since $\hat{L}$ is strictly convex.

Definition 2.3. The efficiency of a driver $\boldsymbol{Y}$ for a prescribed momentum vector $\boldsymbol{P} \in \mathbb{R}^{N}$ is

$$
e(\boldsymbol{P}, \boldsymbol{Y}):=\frac{\Delta(\boldsymbol{P}, \boldsymbol{Y})}{S(\boldsymbol{P}, \boldsymbol{Y})}
$$

In particular, the efficiency of a self-locomotive driver is

$$
e(0, \boldsymbol{Y}):=\frac{\Delta(0, \boldsymbol{Y})}{S(0, \boldsymbol{Y})}
$$

Lemma 2.3. For any mechanical system L, mobilizing driver $\boldsymbol{Y}$ and momentum vector $\boldsymbol{P}$,

$$
0<e(\boldsymbol{P}, \boldsymbol{Y})<1
$$

Proof. By (2.8) and Definition 2.2 it follows that

$$
\Delta(\boldsymbol{P}, \boldsymbol{Y})=\hat{L}\left(\frac{\Delta_{\boldsymbol{Y}} \boldsymbol{X}}{T}, \mathbf{0}\right)-\boldsymbol{P} \cdot \frac{\Delta_{\boldsymbol{Y}} \boldsymbol{X}}{T}+\hat{H}(\boldsymbol{P}, \mathbf{0})
$$

We prove now that

$$
-\frac{1}{T} \int_{0}^{T} \hat{H}(\boldsymbol{P}, \dot{\boldsymbol{Y}}) \mathrm{d} t>\hat{L}\left(\frac{\Delta_{\boldsymbol{Y}} \boldsymbol{X}}{T}, \mathbf{0}\right)-\boldsymbol{P} \cdot \frac{\Delta_{\boldsymbol{Y}} \boldsymbol{X}}{T} .
$$

The left of (2.11) is nothing but

$$
\frac{1}{T} \int_{0}^{T}[\hat{L}(\dot{\boldsymbol{X}}, \dot{\boldsymbol{Y}})-\boldsymbol{P} \cdot \dot{\boldsymbol{X}}] \mathrm{d} t=\frac{1}{T} \int_{0}^{T} \hat{L}(\dot{\boldsymbol{X}}, \dot{\boldsymbol{Y}}) \mathrm{d} t-\boldsymbol{P} \cdot \frac{\Delta_{\boldsymbol{Y}} \boldsymbol{X}}{T},
$$

where $\boldsymbol{X}$ is the optimal solution corresponding to the driver $\boldsymbol{Y}$. So, we only have to prove that

$$
\frac{1}{T} \int_{0}^{T} \hat{L}(\dot{\boldsymbol{X}}, \dot{\boldsymbol{Y}}) \mathrm{d} t>\hat{L}\left(\frac{\Delta_{\boldsymbol{Y}} \boldsymbol{X}}{T}, \mathbf{0}\right)
$$

The above is nothing but Jensen's inequality, which can we written as

$$
\frac{1}{T} \int_{0}^{T} \hat{L}(\dot{\boldsymbol{X}}, \dot{\boldsymbol{Y}}) \mathrm{d} t \geq \hat{L}\left(\frac{1}{T} \int_{0}^{T} \dot{\boldsymbol{X}} \mathrm{d} t, \frac{1}{T} \int_{0}^{T} \dot{\boldsymbol{Y}} \mathrm{d} t\right)=\hat{L}\left(\frac{\Delta_{\boldsymbol{Y}} X}{T}, \mathbf{0}\right)
$$


where we used the definition of $\Delta_{\boldsymbol{Y}} \boldsymbol{X}$ and (2.4) in the last step. Finally, since $\hat{L}$ is strictly convex, the equality above holds if and only if $\dot{\boldsymbol{Y}}=0$ and $\dot{\boldsymbol{X}}=\Delta_{\boldsymbol{Y}} \boldsymbol{X} / T$, which is possible only if $\boldsymbol{Y}$ is not mobilizing for the given $\boldsymbol{P}$.

Definition 2.4. Given a convex Lagrangian $L$ satisfying $(2.1,2.2)$ and $\boldsymbol{P} \in \mathbb{R}^{N}$,

$$
\bar{e}(\boldsymbol{P}):=\sup _{\boldsymbol{Y}} e(\boldsymbol{P}, \boldsymbol{Y}) .
$$

where the supremum is taken over all $T$-periodic, mobilizing $\boldsymbol{Y}$ satisfying (2.4).

\section{Special Case: homogeneous Lagrangians}

We now pose the following problem:

(1) Is $\bar{e}(\boldsymbol{P})=1$ ?

(2) If $\bar{e}(\boldsymbol{P})<1$, is the supremum in $(2.12)$ achieved?

To handle these problems we find it convenient to pose some assumptions on the Lagrangian function.

\subsection{Basic definitions}

Let us concentrate on the special case where the Lagrangian corresponds to a set of mutually independent $n$ particles. The Lagrangian function we consider is

$$
L\left(\dot{q}_{1}, \ldots, \dot{q}_{n}\right):=\sum_{1}^{n}\left|\dot{q}_{i}\right|^{\sigma}
$$

where $\sigma>1$.

We further restrict ourselves to a single driver, so $N=1$ and $\hat{L}: \mathbb{R}^{1} \times \mathbb{R}^{n}$ given by

$$
\hat{L}(\dot{X}, \dot{\boldsymbol{Y}})=\sum_{1}^{n}\left|\dot{X}+\dot{Y}_{i}\right|^{\sigma},
$$

where we use the convention of immovable center of mass

$$
[\boldsymbol{Y}](t):=\sum_{1}^{n} Y_{i}(t) \equiv 0 .
$$

The associated Hamiltonian is, accordingly,

$$
\hat{H}(P, \dot{\boldsymbol{Y}}):=\sup _{W \in \mathbb{R}}\{P W-\hat{L}(W, \dot{\boldsymbol{Y}})\},
$$

so, for the "neutral" driver, the Hamiltonian is

$$
\hat{H}(P, 0)=\sigma^{1 /(\sigma-1)}\left(1-\sigma^{-1}\right) P^{\sigma /(\sigma-1)} .
$$

We shall further assume a unit period $T=1$ and the case $P=0$. In that case, $\Delta_{\mathbf{0}} X=\mathbf{0}$ by (2.8) and Definition 2.4 yields

$$
\Delta(0, \boldsymbol{Y})=\hat{L}\left(\Delta_{\boldsymbol{Y}} X, \mathbf{0}\right)=\left|\Delta_{\boldsymbol{Y}} X\right|^{\sigma} .
$$

Analogously

$$
S(0, \dot{\boldsymbol{Y}})=-\int_{0}^{1} \hat{H}(0, \dot{\boldsymbol{Y}}) \mathrm{d} t .
$$


For later convenience define

$$
U(\dot{\boldsymbol{Y}}):=\frac{\partial \hat{H}}{\partial P}(0, \dot{\boldsymbol{Y}})
$$

We observe that

$$
\Delta_{\boldsymbol{Y}} X=\int_{0}^{1} U(\dot{\boldsymbol{Y}}) \mathrm{d} t
$$

In terms of $U,(3.5)$ and (3.6) take the form

$$
\Delta(0, \boldsymbol{Y})=\left|\int_{0}^{1} U(\dot{\boldsymbol{Y}}) \mathrm{d} t\right|^{\sigma} ; S(0, \boldsymbol{Y})=\int_{0}^{1} \hat{L}(U(\dot{\boldsymbol{Y}}), \dot{\boldsymbol{Y}}) \mathrm{d} t
$$

The first question to be asked is the mobility of this system. Is there a driver $\boldsymbol{Y}$ which mobilizes this Lagrangian at $P=0$ ?

Lemma 3.1. There exists a driver $\boldsymbol{Y}$ which mobilizes the Lagrangian (3.1) if and only if $U$ is not a linear function.

Proof. If $U$ is a linear function, then for any 1 -periodic $\boldsymbol{Y},(3.3)$ implies $\int_{0}^{1} U(\dot{\boldsymbol{Y}}) \mathrm{d} t=0$. Conversely, assume $U$ is not linear. Then there exists a function $\psi: \mathbb{R}^{n} \rightarrow \mathbb{R}^{+}$satisfying

$$
\int_{\mathbb{R}^{n}} \psi(\boldsymbol{v}) \mathrm{d} \boldsymbol{v}=1, \int_{\mathbb{R}^{n}} \boldsymbol{v} \psi(\boldsymbol{v}) \mathrm{d} \boldsymbol{v}=0, \int_{\mathbb{R}^{n}} U(\boldsymbol{v}) \psi(\boldsymbol{v}) \mathrm{d} \boldsymbol{v} \neq 0 .
$$

By the argument below (3.16) there exists $\boldsymbol{Y}:[0,1] \rightarrow \mathbb{R}^{n}$ so that

$$
\int_{0}^{1} U(\dot{\boldsymbol{Y}}) \mathrm{d} t=\int_{\mathbb{R}^{n}} U(\boldsymbol{v}) \psi(\boldsymbol{v}) \mathrm{d} \boldsymbol{v}
$$

In addition, the following property holds for $U$ :

Lemma 3.2. For any $\alpha \in \mathbb{R}^{+}$and $\lambda \in \mathbb{R}$ and $\mathbf{1}=(1, \ldots, 1) \in \mathbb{R}^{n}$, the function $U$ satisfies the following invariance properties:

$$
\forall \boldsymbol{v} \in \mathbb{R}^{n}, \quad U(\alpha \boldsymbol{v})=\alpha U(\boldsymbol{v}) ; \quad U(\boldsymbol{v}+\lambda \mathbf{1})=U(\boldsymbol{v})+\lambda .
$$

Proof. Follows immediately from (3.2) and (3.4) since $\hat{L}(\alpha V, \alpha \boldsymbol{v})=|\alpha|^{\sigma} \hat{L}(V, \boldsymbol{v})$ as well as $\hat{L}(V+\lambda, \boldsymbol{v}+\lambda \mathbf{1})=\hat{L}(V, \boldsymbol{v})$.

Corollary 3.1. If either $\sigma=2$ or $n=2$ then $L$ is not self-locomotive at $P=0$ for any driver $\boldsymbol{Y}$.

Proof. If $\sigma=2$ then $\hat{L}$ is a quadratic function of $X$ and $\hat{H}$ is also quadratic with respect to $P$, with coefficients which are linear in $\dot{\boldsymbol{Y}}$. This implies the result by Lemma 3.1. If $n=2$ then the shift-homogeneity in Lemma 3.2 implies that $U\left(q_{1}, q_{2}\right)=f\left(q_{1}-q_{2}\right)+q_{1}$ for some function $f$ of a single variable. From the scale-homogeneity it follows that

$$
f\left(\alpha\left(y_{1}-y_{2}\right)\right)+\alpha y_{1}=\alpha f\left(y_{1}-y_{2}\right)+\alpha y_{1} \Longrightarrow \alpha f(\zeta)=f(\alpha \zeta)
$$

for any $\alpha \in \mathbb{R}^{+}$and $\zeta \in \mathbb{R}$. Hence, either $f(\zeta)=\gamma \zeta$ or $f(\zeta)=\gamma|\zeta|$ for some $\gamma \in \mathbb{R}$. It is easily shown that $f$ must change sign on $\mathbb{R}$, so it is linear and so is $U$. 


\subsection{Main result}

Let us define the set of all drivers $\boldsymbol{Y}$ corresponding to the homogeneous mechanical system (3.2):

$$
\Lambda_{\sigma}:=\left\{\boldsymbol{Y}:[0,1] \rightarrow \mathbb{R}^{n} ; \int_{0}^{1}|\dot{\boldsymbol{Y}}|^{\sigma}<\infty, \sum_{1}^{n} Y_{i} \equiv 0\right.
$$

Theorem 1. If the homogeneous mechanical system (3.2) is mobilized at $P=0$ then there exists a maximizer of $\bar{e}(\mathbf{0}) \equiv \sup e(0, Y)$ in the set $\Lambda_{\sigma}$.

In particular, by Lemma 2.3:

Corollary 3.2. Under the condition of Theorem $1, \bar{e}(\mathbf{0})<1$.

Remark: Corollary 3.1 implies that $n>2$ and $\sigma \neq 2$ are necessary for the condition of Theorem 1 . We conjecture that these conditions are also sufficient. In any case, it is not difficult to construct examples for $P=0$ mobilized homogeneous systems. For example, take $\sigma=3, n=3$. The function $U=U\left(Y_{1}, Y_{2}, Y_{3}\right)$ can be readily calculated as the root of a quadratic equation whose coefficients are linear functions of $Y_{i}$. The discriminant, however, is not a complete square, so $U$ is not linear.

From Lemma 3.2, (3.7) and the homogeneity of $\hat{L}$ it follows by Definition 2.3 that

$$
e(0, \alpha \boldsymbol{Y})=e(0, Y)
$$

In addition, $e(0, \boldsymbol{Y})$ is clearly invariant under diagonal shifts $\boldsymbol{Y} \rightarrow \boldsymbol{Y}(t)+\beta(t) \mathbf{1}$ where $\mathbf{1}=(1, \ldots 1) \in \mathbb{R}^{n}$. Define now

$$
\Lambda_{\sigma}^{0}:=\left\{\boldsymbol{Y}=\left(Y_{1}, \ldots Y_{n}\right) \in \Lambda_{\sigma} ; \quad Y_{1} \equiv 0 .\right\}
$$

and

$$
\left.S_{\sigma}=\left.\left\{\boldsymbol{Y} \in \Lambda_{\sigma}^{0} ; \quad \int_{0}^{1} \mid \dot{\boldsymbol{Y}}(t)\right)\right|^{\sigma} \mathrm{d} t=1\right\} ; B_{\sigma}=\left\{\boldsymbol{Y} \in \Lambda_{\sigma}^{0} ; \quad \int_{0}^{1}|(\dot{\boldsymbol{Y}}(t))|^{\sigma} \mathrm{d} t \leq 1\right\} .
$$

It follows that

$$
\bar{e}(0):=\sup _{\boldsymbol{Y} \in \Lambda_{\sigma}-\{0\}} e(0, \boldsymbol{Y})=\sup _{\boldsymbol{Y} \in S_{\sigma}} e(0, \boldsymbol{Y})=\sup _{\boldsymbol{Y} \in B_{\sigma}-\{0\}} e(0, \boldsymbol{Y}) .
$$

Let now $\boldsymbol{Y}_{j}$ be a maximizing sequence of $e(0)$ in $S_{\sigma}$. There is a weak limit $\boldsymbol{Y}_{\infty} \in B_{\sigma}$ of this sequence. The inequality

$$
\lim _{j \rightarrow \infty} \Delta\left(0, \boldsymbol{Y}_{j}\right) \leq \Delta\left(0, \boldsymbol{Y}_{\infty}\right)
$$

holds since $\Delta(0, \boldsymbol{Y})$ is upper-semi-continuous, but we do not have the same claim for $S(0, \boldsymbol{Y})$. So, we cannot prove that $\boldsymbol{Y}_{\infty}$ is a maximizer of $e$ in $B_{\sigma}$.

Another problem is that we may have $\boldsymbol{Y}_{\infty}=\mathbf{0}$, so $e\left(0, \boldsymbol{Y}_{\infty}\right)$ is not defined at all. As an example, let $\boldsymbol{Y} \in \Lambda_{\sigma}$ and assume that $\int_{0}^{1} U(\dot{\boldsymbol{Y}}) \neq 0$. This, in particular, implies that $X(t):=\int^{t} U(\dot{\boldsymbol{Y}})=\tilde{X}(t)+\lambda t$ where $\tilde{X}$ is a periodic function and $\lambda \neq 0$. If we replace $\boldsymbol{Y}(t)$ by $\boldsymbol{Y}_{j}=\boldsymbol{Y}_{j}(t):=j^{-1} \boldsymbol{Y}(j t)$ for $j \in \mathbb{N}$, using the periodicity of $\boldsymbol{Y}$ to define $\boldsymbol{Y}_{j}$ on $(0,1)$, the following claims are straightforward:

(1) $\boldsymbol{Y}_{j} \in \Lambda_{\sigma}$ for any $j \in \mathbb{N}$.

(2) $S\left(0, \boldsymbol{Y}_{j}\right)=S(0, \boldsymbol{Y})$.

(3) $\Delta_{\boldsymbol{Y}_{j}} \boldsymbol{X}=\Delta_{\boldsymbol{Y}} \boldsymbol{X}$, so $\Delta\left(0, \boldsymbol{Y}_{j}\right)=\Delta(0, \boldsymbol{Y})$.

(4) $\lim _{j \rightarrow \infty} \dot{\boldsymbol{Y}}_{j}=0$ weakly.

In particular, we find out that $e\left(0, \boldsymbol{Y}_{j}\right)=e(0, \boldsymbol{Y})$, while $e(0, \cdot)$ is not defined for the weak $\operatorname{limit}_{\lim } \rightarrow \infty \dot{\boldsymbol{Y}}_{j}=\mathbf{0}$. 


\subsection{Relaxation}

To overcome the last difficulty we shall extend Definition 2.3 as follows: Let

$$
\mathcal{P}^{n}:=\left\{\text { Probability Borel measures on } \mathbb{R}^{n}\right\} .
$$

Given $\nu \in \mathcal{P}^{n}$, let

$$
\begin{aligned}
\Delta_{\nu} X & :=\int_{\mathbb{R}^{n}} U(\boldsymbol{V}) \nu(\mathrm{d} \boldsymbol{V}) . \\
\langle\hat{H}(P)\rangle_{\nu} & :=\int_{\mathbb{R}^{n}} \hat{H}(P, \boldsymbol{V}) \nu(\mathrm{d} \boldsymbol{V}) .
\end{aligned}
$$

The relaxation of Definition 2.3 for $P=0$ is given by

$$
\langle e(0)\rangle_{\nu}:=-\frac{\left|\Delta_{\nu} X\right|^{\sigma}}{\langle\hat{H}(0)\rangle_{\nu}}=\frac{\left|\int U(\boldsymbol{V}) \nu(\mathrm{d} \boldsymbol{V})\right|^{\sigma}}{\int_{\mathbb{R}^{n}} \sum_{1}^{n}\left|V_{i}+U(\boldsymbol{V})\right|^{\sigma} \nu(\mathrm{d} \boldsymbol{V})} .
$$

We shall further extend the definitions of $\Lambda_{\sigma}$ and $\Lambda_{\sigma}^{0}$ as follows:

$$
\begin{gathered}
\bar{\Lambda}_{\sigma}:=\left\{\nu=\nu(\mathrm{d} \boldsymbol{V}) \in \mathcal{P}^{n} ; \int_{\mathbb{R}^{n}}|\boldsymbol{V}|^{\sigma} \nu(\mathrm{d} \boldsymbol{V})<\infty \quad ; \quad \int_{\mathbb{R}^{n}} V_{i} \nu(\mathrm{d} \boldsymbol{V})=0,1 \leq i \leq n\right\} \\
\bar{\Lambda}_{\sigma}^{0}:=\left\{\nu \in \bar{\Lambda}_{\sigma} ; \nu=\delta_{V_{1}} \otimes \mu\left(\mathrm{d} V_{2}, \ldots, \mathrm{d} V_{n}\right) \text { where } \mu \in \mathcal{P}^{n-1}\right\} .
\end{gathered}
$$

Lemma 3.3. For any $\boldsymbol{Y} \in \Lambda_{\sigma}$ (res. $\left.\boldsymbol{Y} \in \Lambda_{\sigma}^{0}\right)$ there exists $\nu \in \bar{\Lambda}_{\sigma}$ (res. $\left.\nu \in \bar{\Lambda}_{\sigma}^{0}\right)$ so that $\langle e(0)\rangle_{\nu}=e(0, \boldsymbol{Y})$. Conversely, for any $\nu \in \bar{\Lambda}_{\sigma}$ (res. $\nu \in \bar{\Lambda}_{\sigma}^{0}$ ) there exists $\boldsymbol{Y} \in \Lambda_{\sigma}$ (res. $\boldsymbol{Y} \in \Lambda_{\sigma}^{0}$ ) so that $\langle e(0)\rangle_{\nu}=e(\boldsymbol{Y}, 0)$.

Remark: The measure $\nu$ associated with $\dot{\boldsymbol{Y}}$ is related to Young measure. In general, however, Young measures are used to study the oscillatory behavior of a weak limit of $\mathbb{L}^{\infty}$ sequences (see, e.g., [5]).

Proof. For the first part, define $\nu(\mathrm{d} \boldsymbol{V})=\int_{0}^{1} \prod_{1}^{n} \delta_{V_{i}-\dot{Y}_{i}(t)}$. To elaborate, the measure $\nu$ corresponding to $\boldsymbol{Y}$ is obtained by its application on test functions $\phi \in C_{0}\left(\mathbb{R}^{n}\right)$ :

$$
\int_{\mathbb{R}^{n}} \phi(\boldsymbol{V}) \nu(\mathrm{d} \boldsymbol{V})=\int_{0}^{1} \phi(\dot{\boldsymbol{Y}}(t)) \mathrm{d} t
$$

The above equality extend to $\phi(s)=s_{i}, i=1 \ldots n$ and $\phi(s)=|s|^{\sigma}$. In particular

$$
\int_{\mathbb{R}^{n}} \boldsymbol{V} \nu(\mathrm{d} \boldsymbol{V})=\int_{0}^{1} \dot{\boldsymbol{Y}}(t) \mathrm{d} t=0 ; \quad \int_{\mathbb{R}^{n}}|\boldsymbol{V}|^{\sigma} \nu(\mathrm{d} \boldsymbol{V})=\int_{0}^{1}|\dot{\boldsymbol{Y}}|^{\sigma}(t) \mathrm{d} t<\infty .
$$

As a result

and $\langle e(0)\rangle_{\nu}=e(\boldsymbol{Y}, 0)$.

$$
\langle\hat{H}(0)\rangle_{\nu}=\int_{0}^{1} \hat{H}(0, \dot{\boldsymbol{Y}}) \mathrm{d} t \quad ; \quad\langle U\rangle_{\nu}=\int_{0}^{1} U(\dot{\boldsymbol{Y}}) \mathrm{d} t
$$

For the second part we use Theorem 2 of [7] to observe the following:

For any such $\nu$ there exists a Borel function $\mathbb{T}:[0,1] \rightarrow \mathbb{R}^{n}$ which push forward the Lebesgue measure $\mathrm{d} t$ on $[0,1]$ to $\nu$. That is, for any test function $\phi \in C_{0}\left(\mathbb{R}^{n}\right)$,

$$
\int_{0}^{1} \phi(\mathbb{T}(t)) \mathrm{d} t=\int_{\mathbb{R}^{n}} \phi(\boldsymbol{V}) \nu(\mathrm{d} \boldsymbol{V})
$$


So, set $\boldsymbol{Y}(t):=\int^{t} \mathbb{T}(s) \mathrm{d} s$. It is absolutely continuous function satisfying $\dot{\boldsymbol{Y}}=\mathbb{T}$ a.e. The definition (3.14) implies also that $\int_{0}^{1}|\dot{\boldsymbol{Y}}|^{\sigma}=\int|v|^{\sigma} \nu(\mathrm{d} \boldsymbol{V})<\infty$, as well as $\int_{0}^{1} \dot{\boldsymbol{Y}} \mathrm{d} t=\int \boldsymbol{V} \nu(\mathrm{d} \boldsymbol{V})=0$, which yields the periodicity of $\boldsymbol{Y}$. The equality (3.16) holds under this identification as well.

From Lemma 3.3 and the definition (3.10) of $\bar{e}(\mathbf{0})$ we obtain:

\section{Corollary 3.3.}

$$
\bar{e}(0)=\sup _{\nu \in \bar{\Lambda}_{\sigma}}\langle e(0)\rangle_{\nu}=\sup _{\nu \in \bar{\Lambda}_{\sigma}^{0}}\langle\boldsymbol{e}(0)\rangle_{\nu}
$$

\subsection{Proof of Theorem 1}

Define now

$$
\psi(\boldsymbol{V}):=|U(\boldsymbol{V})|+\sum_{i=2}^{N}\left|V_{i}+U(\boldsymbol{V})\right|, \boldsymbol{V}=\left(0, V_{2}, \ldots, V_{N}\right) .
$$

We shall summertime some properties of $\psi$ which will be needed later:

Lemma 3.4. There exists $A>0$ so that

$$
A^{-1}|\boldsymbol{V}| \leq|\psi(\boldsymbol{V})| \leq A|\boldsymbol{V}| \quad \forall \boldsymbol{V}=\left(0, V_{2}, \ldots, V_{N}\right) .
$$

In addition, for any $\nu \in \bar{\Lambda}_{\sigma}^{0}$,

$$
A^{-1} \int_{\mathbb{R}^{N}}|\psi(\boldsymbol{V})|^{\sigma} \nu(\mathrm{d} \boldsymbol{V})<\langle\hat{H}(0)\rangle_{\nu} \leq A \int_{\mathbb{R}^{N}}|\psi(\boldsymbol{V})|^{\sigma} \nu(\mathrm{d} \boldsymbol{V}) .
$$

Proof. The estimate (3.18) follows from the homogeneity of $U$, namely $U(\alpha \boldsymbol{V})=\alpha U(\boldsymbol{V})$, as well as from the evident property $U(0, V, \ldots, V)=0 \Longleftrightarrow V=0$. The estimate (3.19) follows from (3.12), as well as (3.18).

Given $1<q<\sigma$, let $\bar{S}_{\sigma}^{q}$ be the unit sphere (resp. $\bar{B}_{\sigma}^{q}$ the unit ball) defined by

$$
\bar{S}_{\sigma}^{q}=\left\{\nu \in \bar{\Lambda}_{\sigma}^{0} ; \quad \int_{\mathbb{R}^{N-1}}|\boldsymbol{V}|^{q} \nu(\mathrm{d} \boldsymbol{V})=1\right\} \quad ; \quad \bar{B}_{\sigma}^{q}=\left\{\nu \in \bar{\Lambda}_{\sigma}^{0} ; \quad \int_{\mathbb{R}^{N-1}}|\boldsymbol{V}|^{q} \nu(\mathrm{d} \boldsymbol{V}) \leq 1\right\} .
$$

Next,

Lemma 3.5. $\bar{e}(0)=\sup _{\nu \in \bar{S}_{\sigma}^{q}}\langle e(0)\rangle_{\nu}$.

Proof. By Corollary 3.3 we only have to show that any $\nu \in \bar{\Lambda}_{\sigma}$ (resp. $\nu \in \bar{\Lambda}_{\sigma}^{0}$ ) can be transformed into $\hat{\nu} \in \bar{S}_{\sigma}$ (resp. $\left.\hat{\nu} \in \bar{S}_{\sigma}^{0}\right)$ so that $\langle e(0)\rangle_{\nu}=\langle e(0)\rangle_{\tilde{\nu}}$. Let $\hat{\nu}(\mathrm{d} \boldsymbol{V})=\beta^{N} \nu(\beta \mathrm{d} \boldsymbol{V})$ for $\beta>0$. The homogeneity properties (3.8) imply that, indeed, $\langle e(0)\rangle_{\nu}=\langle e(0)\rangle_{\tilde{\nu}}$. In addition, $\int \boldsymbol{V} \hat{\nu}(\mathrm{d} \boldsymbol{V})=\beta^{-1} \int \boldsymbol{V} \nu(\mathrm{d} \boldsymbol{V})=0$ if $\nu \in \bar{\Lambda}_{\sigma}$. Moreover, $\int|\boldsymbol{V}|^{q} \hat{\nu}(\mathrm{d} \boldsymbol{V})=\beta^{-q} \int|\boldsymbol{V}|^{q} \nu(\mathrm{d} \boldsymbol{V})$, so $\hat{\nu} \in \bar{S}_{\sigma}$ if $\beta=\left(\int|\boldsymbol{V}|^{q} \nu(\mathrm{d} \boldsymbol{V})\right)^{1 / q}$.

Let $\nu_{j} \in \bar{S}_{\sigma}^{q}$ be a maximizing sequence of $\bar{e}(0)$, according to Lemma 3.5. Since the $q>1$ moments of $\nu_{j}$ are uniformly bounded, this sequence is compact (tight) in the weak topology of measures. Let $\nu_{\infty}$ be the weak limit of $\nu_{j}$. Since $|U(\boldsymbol{V})| \leq A|\boldsymbol{V}|$ for some $A>0$ it follows that the sequence of (signed) measures $U(\boldsymbol{V}) \nu_{j}(\mathrm{~d} \boldsymbol{V})$ is tight as well, and that

$$
\lim _{j \rightarrow \infty} U(\boldsymbol{V}) \nu_{j}(\mathrm{~d} \boldsymbol{V})=U(\boldsymbol{V}) \nu_{\infty}(\mathrm{d} \boldsymbol{V}) .
$$

On the other hand, it is not a-priori evident that $\nu_{\infty} \in \bar{S}_{\sigma}^{q}$, since the sequence $|\boldsymbol{V}|^{q} \nu_{j}(\mathrm{~d} \boldsymbol{V})$ is not necessarily tight, so we only know $\nu_{\infty} \in \bar{B}_{\sigma}^{q}$, namely

$$
\int|\boldsymbol{V}|^{q} \nu_{\infty}(\mathrm{d} \boldsymbol{V}) \leq 1
$$


We claim, however, that, in fact, the $\sigma$ moments of $\nu_{j}$ are uniformly bounded. For, if $\int|\boldsymbol{V}|^{\sigma} \nu_{j}(\mathrm{~d} \boldsymbol{V}) \rightarrow \infty$, then by $(3.18,3.19)$ and $(3.12)$ also $-\langle\hat{H}(0)\rangle_{\nu_{j}} \rightarrow \infty$. Since we also know that $\langle U\rangle_{\nu_{j}}$ are uniformly bounded, then

$$
\langle e(0)\rangle_{\nu_{j}}=-\frac{\langle U\rangle_{\nu_{j}}}{\langle\hat{H}(0)\rangle_{\nu_{j}}} \rightarrow 0,
$$

contradicting the assumption that $\nu_{j}$ is a maximum sequence for $\bar{e}(0)$.

Since $q<\sigma$ by assumption it follows that the sequence $|\boldsymbol{V}|{ }^{q} \nu_{j}(\mathrm{~d} \boldsymbol{V})$ is tight as well, so there is, in fact, an equality in $(3.22)$ and

$$
\nu_{\infty} \in \bar{S}_{\sigma}^{q} .
$$

In addition, for any non-negative continuous function $\phi$ on $\mathbb{R}^{n}$

$$
\liminf _{j \rightarrow \infty} \int_{\mathbb{R}^{n}} \phi(\boldsymbol{V}) \nu_{j}(\mathrm{~d} \boldsymbol{V}) \geq \int_{\mathbb{R}^{n}} \phi(\boldsymbol{V}) \nu_{\infty}(\mathrm{d} \boldsymbol{V}) .
$$

In particular

$$
\liminf _{j \rightarrow \infty}\left[-\langle\hat{H}(0)\rangle_{\nu_{j}}\right] \equiv \liminf _{j \rightarrow \infty} \int_{\mathbb{R}^{n}} \sum_{i=1}^{N}\left|V_{i}+U(\boldsymbol{V})\right|^{\sigma} \nu_{j}(\mathrm{~d} \boldsymbol{V}) \geq-\langle\hat{H}(0)\rangle_{\nu_{\infty}}
$$

As a result

$$
\limsup _{j \rightarrow \infty}\langle e(0)\rangle_{\nu_{j}} \leq\langle e(0)\rangle_{\nu_{\infty}} .
$$

Since $\nu_{j}$ is a maximizing sequence of $\bar{e}(0)$ and (3.23) holds, there must be an equality in (3.24) and Theorem 1 follows.

\section{Conclusions}

We proved the existence of a mobilizing driver (in particular, of self locomotion) for some mechanical systems in an Euclidian space. This seems to contradict the geometrical theory of self locomotion as developed in [1]. The later requires the existence of curvature in the ambient space.

Let us consider the role of self locomotion played by the geometry. A shift (isometry) of a body is generated by a Killing vector field $\xi$. A Killing vector filed satisfies the Killing equation

$$
\nabla_{(j} \xi_{k)}:=\nabla_{j} \xi_{k}+\nabla_{k} \xi_{j}=0
$$

which means that it generates no strain [9]. A deformation field $\eta$, on the other hand, generates a strain

$$
\Sigma(\eta)=\nabla_{(j} \eta_{k)} .
$$

A body (swimmer) may control the strain but not directly the deformation filed on its own body. But, for a given strain $\Sigma$, the deformation is determined (and (4.1) can be solved) only up to an arbitrary Killing vector field $\xi$. At this point the Riemannian structure marshes in: The deformation is uniquely defined by the condition that it is perpendicular (with respect to the underlying inner product), to the space of all Killing vector fields on the manifold

$$
\langle\eta, \xi\rangle=0
$$

where $\langle$,$\rangle is the inner product. The infinitesimal shift of the body \delta \tau$ due to a deformation $\eta d \sigma$ subjected to (4.2) is given by (see (4.7) in [1])

$$
\langle\xi, \xi\rangle \delta \tau=-\frac{1}{2}\left\langle\mathrm{~d} \xi, \eta_{a} \eta_{b}\right\rangle \mathrm{d} \sigma^{b} \mathrm{~d} \sigma^{a} .
$$

Thus, a necessary condition for self locomotion is that the curvature 2-form $\mathrm{d} \xi$ of the Killing field is non zero. 
In our case, there is no Riemannian structure, as we assume a non-quadratic Lagrangian. However, we utilize the Euclidian structure, which allow a unique center of mass, to separate the shift from the deformation. We do it by the gauge assumption (2.4), which impose a rest state for the center of mass of the drivers. The result shows that self locomotion is possible if the Lagrangian is non-quadratic (i.e, the geometry is non-Riemannian). Another necessary condition for self-locomotion is that the system contains at least 3 particles. This is, again, in contrast to the geometric theory, which allows "swimmers" of only 2 particles (Sect. A of [1]).

In addition to the generalized notion of drivers and self locomotion discussed in this paper, we also introduced a natural definition of efficiency. This is a measure for the relation between the shift made by driver per cycle and the energy applied to execute this shift. We showed that, under the stated conditions, a "best driver", which realizes the maximal possible efficiency, does exist.

\section{REFERENCES}

[1] J.E. Avron and O. Kenneth, Swimming in curved space or The Baron and the Cat. Arxiv preprint math-ph/0602053 - arxiv.org (2006).

[2] D. Chipot, M. Kinderlehrer and M. Kowalczyk, A variational principle for molecular motors, dedicated to piero villagio on the occasion of his 60 birthday. Mechanica 38 (2003) 505-518.

[3] S. Chipot, M. Hastings and D. Kinderlehrer, Transport in a molecular system. ESAIM: M2AN 38 (2004) 1011-1034.

[4] D. Dolbeault, J. Kinderlehrer and M. Kowalczyk, Remarks about the flashing rachet, partial differential equations and inverse problems. Contemp. Math. 362 (2004) 167-175.

[5] L.C. Evans, Weak Convergence Methods for Nonlinear Partial Differential Equations. CEMS 74, American Mathematical Society (1990).

[6] O. Gat, J.E. Avron and O. Kenneth, Optimal swimming at low reynolds numbers. Phys. Rev. Lett. 93 (2004) 18.

[7] J.C. Oxtoby, Homeomorphic measures in metric spaces. Proc. Amer. Math. Soc. 24 (1970) 419-423.

[8] R.E. Raspe, The Surprising Adventures of Baron Munchusen. Kessinger Publishing (2004).

[9] R.M. Wald, General Relativity, Appendix C3. The University of Chicago Press (1984).

[10] J. Wisdom, Swimming in sapcetime: Motion by cyclic changes in body shape. Science 299 (2003) 1865-1869. 